Теорія Ймовір. та Матем. Статист. Вип. 79, 2008
Theor. Probability and Math. Statist.

No. 79, 2009, Pages 143-151 S 0094-9000(09)00788-1

Article electronically published on December 30, 2009

\title{
PARAMETRIC ESTIMATION FOR LINEAR SYSTEM OF STOCHASTIC DIFFERENTIAL EQUATIONS DRIVEN BY FRACTIONAL BROWNIAN MOTIONS WITH DIFFERENT HURST INDICES
}

UDC 519.21

\author{
B. L. S. PRAKASA RAO
}

\begin{abstract}
We consider the problem of maximum likelihood estimation of the common trend parameter for a linear system of stochastic differential equations driven by two independent fractional Brownian motions possibly with different Hurst indices. Asymptotic properties of the maximum likelihood estimator are discussed.
\end{abstract}

\section{INTRODUCTION}

Statistical inference for diffusion type processes satisfying stochastic differential equations driven by a Wiener process is now well known [6. There has been some recent interest to study similar problems for stochastic processes driven by fractional Brownian motion (fBm) in view of their applications to modelling of internet traffic and study of fluctuation in market share with long range dependence. Properties of maximum likelihood estimators for linear stochastic differential equations driven by fractional Brownian motion (fBm) are investigated in [1] and [8, 9, 10]. Geometric Brownian motion has been widely used for modelling fluctuations of share prices in the stock market, and geometric fractional Brownian motion, that is, a process governed by a stochastic differential equation of the type

$$
d X(t)=\theta X(t) d t+\sigma_{1} X(t) d W^{h}(t), \quad X(0)=x_{0} \in \mathbb{R}, 0 \leq t \leq T
$$

has also been studied as a model for modelling fluctuations in share prices in the stock market in the presence of long range dependence. In the present scenario where the fluctuations in share prices in one country are influenced by the same in another country or within the same country from different regions, it is reasonable to model the share prices by a system of stochastic differential equations driven by noise components coming from different environments which could be dependent or independent.

We discuss estimation of the trend for a linear system of stochastic differential equations in Section 2 and specialize the results to a linear system of geometric fractional Brownian motion in Section 3.

2000 Mathematics Subject Classification. Primary 62M09; Secondary 60G18. 


\section{General CASE}

Let $(\Omega, \mathcal{F}, \mathrm{P})$ be a complete probability space with a filtration $\left\{\mathcal{F}_{t}, t \geq 0\right\}$. Consider the linear stochastic differential system

$$
\begin{gathered}
d X(t)=\theta a_{1}(t, X(t)) d t+b_{1}(t, X(t)) d W^{H}(t), \quad X(0)=x_{0} \in \mathbb{R}, 0 \leq t \leq T_{1}, \\
d Y(t)=\theta a_{2}(t, Y(t)) d t+b_{2}(t, Y(t)) d W^{h}(t), \quad Y(0)=y_{0} \in \mathbb{R}, 0 \leq t \leq T_{2},
\end{gathered}
$$

where $\theta \in \Theta \subset \mathbb{R} \backslash\{0\}$. The functions $b_{1}$ and $b_{2}$ are assumed to be known and nonvanishing. We further assume that the functions $a_{1}$ and $a_{2}$ are also known and that the fractional Brownian motions $\left\{W^{h}(t), 0 \leq t \leq T\right\}$ and $\left\{W^{H}(t), 0 \leq t \leq T\right\}$ with known Hurst indices $h \in\left[\frac{1}{2}, 1\right)$ and $H \in\left[\frac{1}{2}, 1\right)$ are independent and adapted to the filtration $\left\{\mathcal{F}_{t}, t \geq 0\right\}$. The latter can be achieved if necessary by choosing $\mathcal{F}_{t}$ to be the $\sigma$-algebra generated by the family

$$
\left\{W^{h}(s), 0 \leq s \leq t ; W^{H}(s), 0 \leq s \leq t\right\} .
$$

We use pathwise construction of the stochastic integral with respect to the fBm discussed in 12. Suppose that system (2.1) has a unique pathwise solution

$$
\left\{X(s), 0 \leq s \leq T_{1} ; Y(s), 0 \leq s \leq T_{2}\right\} .
$$

Sufficient conditions for the existence and uniqueness of the solution are given in [5]. In addition to these conditions, we assume that

$$
\frac{a_{i}(t, X(t))}{b_{i}(t, X(t))}
$$

is Lebesgue integrable on $\left[0, T_{i}\right], i=1,2$. Let $P_{t}^{Z}$ be the measure generated by a process $Z$ on $[0, t]$. We will now calculate the Radon-Nikodym derivative for probability measures $Q$ on $(\Omega, \mathcal{F})$ such that $P_{t}^{X}$ is equivalent to $Q_{t}^{X}, 0 \leq t \leq T_{1}$, and the process $X$ has zero drift under the measure $Q$. Let

$$
\phi_{t} \equiv \psi(t, X(t))=-\theta \frac{a_{1}(t, X(t))}{b_{1}(t, X(t))} .
$$

Define, for $0<s<t \leq T_{1}$,

$$
k_{H}(t, s)= \begin{cases}\kappa_{H}^{-1} s^{1 / 2-H}(t-s)^{1 / 2-H}, & 0 \leq s \leq t, \\ 0, & \text { otherwise }\end{cases}
$$

where

$$
\kappa_{H}=2 H \Gamma\left(\frac{3}{2}-H\right) \Gamma\left(H+\frac{1}{2}\right) .
$$

Suppose $\psi(t, x) \in C^{1,2}\left(\left[0, T_{1}\right] \times \mathbb{R}\right)$. Then, by [3, Lemma 1], there exists another $\mathcal{F}_{t^{-}}$ predictable process $\left\{\delta_{s}, 0 \leq s \leq T_{1}\right\}$ such that

$$
\int_{0}^{t} \delta_{s} d s<\infty \quad \mathrm{P} \text {-a.s., } \quad 0 \leq t \leq T_{1},
$$

and

$$
J_{t}:=\int_{0}^{t} k_{H}(t, s) \phi_{s} d s=\int_{0}^{t} \delta_{s} d s, \quad 0 \leq t \leq T_{1} .
$$

It is proved in 4] that

$$
\int_{0}^{t} k_{H}(t, s) d W_{s}^{H}=\int_{0}^{t} s^{1 / 2-H} d \widetilde{W}_{s}, \quad 0 \leq t \leq T_{1},
$$


where the stochastic integral on the left side exists as a pathwise integral with respect to the $\mathrm{fBm} W^{H}$,

$$
\widetilde{W}_{t}=\int_{0}^{t} s^{H-1 / 2} d \widetilde{M}_{s}, \quad 0 \leq t \leq T_{1}
$$

and

$$
\widetilde{M}_{t}=\int_{0}^{t} k_{H}(t, s) d W_{s}^{H}, \quad 0 \leq t \leq T_{1} .
$$

Furthermore the process $\left\{\widetilde{W}_{s}, 0 \leq t \leq T_{1}\right\}$ is a standard Wiener process. Suppose that

$$
\mathrm{E}\left(\int_{0}^{T_{1}} s^{2 H-1} \delta_{s}^{2} d s\right)<\infty
$$

Define

$$
\widetilde{L}_{t}=\int_{0}^{t} s^{H-1 / 2} \delta_{s} d \widetilde{W}_{s}, \quad 0 \leq t \leq T_{1} .
$$

Under assumption (2.6), the process $\left\{\widetilde{L}_{t}, \mathcal{F}_{t}, 0 \leq t \leq T_{1}\right\}$ is a square integrable martingale. Suppose the martingale $\left\{\widetilde{L}_{t}, \mathcal{F}_{t}, 0 \leq t \leq T_{1}\right\}$ satisfies the condition

$$
\mathrm{E}\left[\exp \left\{\widetilde{L}_{t}-\frac{1}{2}\langle\widetilde{L}\rangle_{t}\right\}\right]=1, \quad 0 \leq t \leq T_{1}
$$

Then it is known that the process

$$
B_{t}^{H}=W_{t}^{H}-\int_{0}^{t} \phi_{s} d s, \quad 0 \leq t \leq T_{1}
$$

is an $\mathrm{fBm}$ with respect to the probability measure $Q_{H}$ defined on $(\Omega, \mathcal{F})$ by

$$
\left.\frac{d Q_{H}}{d \mathrm{P}}\right|_{\mathcal{F}_{t}}=\exp \left\{\widetilde{L}_{t}-\frac{1}{2}\langle\widetilde{L}\rangle_{t}\right\}, \quad 0 \leq t \leq T_{1}
$$

Note that

$$
\left.\frac{d Q_{H}}{d \mathrm{P}}\right|_{\mathcal{F}_{t}}=\exp \left\{\int_{0}^{t} s^{H-1 / 2} \delta_{s} d \widetilde{W}_{s}-\frac{1}{2} \int_{0}^{t} s^{2 H-1} \delta_{s}^{2} d s\right\}, \quad 0 \leq t \leq T_{1} .
$$

Analogously to the above discussion, we construct another probability measure $Q_{h}$ defined on $(\Omega, \mathcal{F})$ such that

$$
\left.\frac{d Q_{h}}{d \mathrm{P}}\right|_{\mathcal{F}_{t}}=\exp \left\{\int_{0}^{t} s^{h-1 / 2} \psi_{s} d \widehat{W}_{s}-\frac{1}{2} \int_{0}^{t} s^{2 h-1} \psi_{s}^{2} d s\right\}, \quad 0 \leq t \leq T_{2} .
$$

Here $\widehat{W}$ is the Wiener process corresponding to the $\mathrm{fBm} W^{h}$, and $\left\{\psi_{t}, 0 \leq t \leq T_{2}\right\}$ and $\left\{\eta_{t}, 0 \leq t \leq T_{2}\right\}$ are processes such that

$$
I_{t}:=\int_{0}^{t} k_{h}(t, s) \eta_{s} d s=\int_{0}^{t} \psi_{s} d s, \quad 0 \leq t \leq T_{2} .
$$

Observe that

$$
B_{t}^{h}=W_{t}^{h}-\int_{0}^{t} \eta_{s} d s, \quad 0 \leq t \leq T_{2},
$$

is an $\mathrm{fBm}$ with respect to the probability measure $Q_{h}$ defined by

$$
\begin{gathered}
\left.\frac{d Q_{h}}{d \mathrm{P}}\right|_{\mathcal{F}_{t}}=\exp \left\{\int_{0}^{t} s^{h-1 / 2} \psi_{s} d \widehat{W}_{s}-\frac{1}{2} \int_{0}^{t} s^{2 h-1} \psi_{s}^{2} d s\right\}, \\
0 \leq t \leq T_{2} .
\end{gathered}
$$


With respect to the probability measures $Q_{H}$ and $Q_{h}$, the trend term should be zero for the first equation in the system over the interval $\left[0, T_{1}\right]$, and it should be zero for the second equation in the system over the interval $\left[0, T_{2}\right]$. Hence

$$
b_{1}(t, X(t)) \phi_{t}=-\theta a_{1}(t, X(t))
$$

and

$$
b_{2}(t, Y(t)) \eta_{t}=-\theta a_{2}(t, Y(t)) .
$$

Observe that (here $g_{t}^{\prime}$ denotes the derivative of $g$ with respect to $t$ evaluated at $t$ )

$$
\begin{aligned}
\frac{d Q}{d \mathrm{P}}= & \left.\left.\frac{d Q_{h}}{d \mathrm{P}}\right|_{\mathcal{F}_{T_{2}}} \frac{d Q_{H}}{d \mathrm{P}}\right|_{\mathcal{F}_{T_{1}}} \\
= & \exp \left\{\int_{0}^{T_{2}} s^{h-1 / 2} J_{s}^{\prime} d \widehat{W}_{s}-\frac{1}{2} \int_{0}^{T_{2}} s^{2 h-1}{J_{s}^{\prime}}^{2} d s\right\} \\
& \times \exp \left\{\int_{0}^{T_{1}} s^{H-1 / 2} I_{s}^{\prime} d \widetilde{W}_{s}-\frac{1}{2} \int_{0}^{T_{1}} s^{2 H-1}{I_{s}^{\prime}}^{2} d s\right\} \\
= & \exp \left\{\int_{0}^{T_{2}} s^{h-1 / 2} J_{s}^{\prime} d \widehat{W}_{s}+\int_{0}^{T_{1}} s^{H-1 / 2} I_{s}^{\prime} d \widetilde{W}_{s}\right. \\
& \left.\quad-\frac{1}{2}\left[\int_{0}^{T_{2}} s^{2 h-1}{J_{s}^{\prime}}^{2} d s+\int_{0}^{T_{1}} s^{2 H-1}{I_{s}^{\prime}}^{2} d s\right]\right\} .
\end{aligned}
$$

Note that

$$
\begin{aligned}
J_{t}^{\prime} & =\left(\int_{0}^{t} k_{h}(t, s) \eta_{s} d s\right)_{t}^{\prime} \\
& =\left(\int_{0}^{t} k_{h}(t, s) \frac{-\theta a_{2}(s, Y(s))}{b_{2}(s, Y(s))} d s\right)_{t}^{\prime} \\
& =-\theta\left(\int_{0}^{t} k_{h}(t, s) \frac{a_{2}(s, Y(s))}{b_{2}(s, Y(s))} d s\right)_{t}^{\prime} \\
& =:-\theta \Delta_{t} .
\end{aligned}
$$

Similarly

$$
I_{t}^{\prime}=-\theta\left(\int_{0}^{t} k_{H}(t, s) \frac{a_{1}(s, X(s))}{b_{1}(s, X(s))} d s\right)_{t}^{\prime}=:-\theta \Gamma_{t} .
$$

From the above relations, we get that

$$
\begin{aligned}
\log \frac{d Q}{d \mathrm{P}}= & \int_{0}^{T_{2}} s^{h-1 / 2} J_{s}^{\prime} d \widehat{W}_{s}+\int_{0}^{T_{1}} s^{H-1 / 2} I_{s}^{\prime} d \widetilde{W}_{s} \\
& -\frac{1}{2}\left[\int_{0}^{T_{1}} s^{2 h-1}{J_{s}^{\prime}}^{2} d s+\int_{0}^{T_{2}} s^{2 H-1}{I_{s}^{\prime}}^{2} d s\right] \\
= & \int_{0}^{T_{2}} s^{h-1 / 2}\left(-\theta \Delta_{s}\right) d \widehat{W}_{s}+\int_{0}^{T_{1}} s^{H-1 / 2}\left(-\theta \Gamma_{s}\right) d \widetilde{W}_{s} \\
& -\frac{1}{2}\left[\int_{0}^{T_{2}} s^{2 h-1}\left(-\theta \Delta_{s}\right)^{2} d s+\int_{0}^{T_{1}} s^{2 H-1}\left(-\theta \Gamma_{s}\right)^{2} d s\right] .
\end{aligned}
$$

In order to estimate the parameter $\theta$ based on the observations of the process

$$
\left\{X(s), 0 \leq s \leq T_{1}\right\}
$$


and of the process $\left\{Y(s), 0 \leq s \leq T_{2}\right\}$, we maximize the function $d Q / d \mathrm{P}$ or equivalently $\log (d Q / d \mathrm{P})$. Differentiating the function $\log (d Q / d \mathrm{P})$ with respect to $\theta$ and equating the derivative to zero, we obtain the likelihood equation

$$
\begin{aligned}
& \theta\left[\int_{0}^{T_{1}} s^{2 H-1} \Gamma_{s}^{2} d s+\int_{0}^{T_{2}} s^{2 h-1} \Delta_{s}^{2} d s\right] \\
& \quad=-\left[\int_{0}^{T_{1}} s^{H-1 / 2} \Gamma_{s} d \widetilde{W}_{s}+\int_{0}^{T_{2}} s^{h-1 / 2} \Delta_{s} d \widehat{W}_{s}\right]
\end{aligned}
$$

The solution $\widehat{\theta}_{T_{1}, T_{2}}$ of this equation is given by

$$
\widehat{\theta}_{T_{1}, T_{2}}=-\frac{\left[\int_{0}^{T_{1}} s^{H-1 / 2} \Gamma_{s} d \widetilde{W}_{s}+\int_{0}^{T_{2}} s^{h-1 / 2} \Delta_{s} d \widehat{W}_{s}\right]}{\left[\int_{0}^{T_{1}} s^{2 H-1} \Gamma_{s}^{2} d s+\int_{0}^{T_{2}} s^{2 h-1} \Delta_{s}^{2} d s\right]},
$$

which is the maximum likelihood estimator in this general case. It can be checked that (see [3, Eq. (14)])

$$
d \widetilde{W}_{s}=d W_{s}^{(1)}-\theta s^{H-1 / 2} \Gamma(s) d s
$$

and

$$
d \widehat{W}_{s}=d W_{s}^{(2)}-\theta s^{h-1 / 2} \Delta(s) d s
$$

where $W^{(1)}$ and $W^{(2)}$ are independent Wiener processes under the measure $Q$. Using these relations, it follows that

$$
\widehat{\theta}_{T_{1}, T_{2}}-\theta=-\frac{\left[\int_{0}^{T_{1}} s^{H-1 / 2} \Gamma_{s} d W_{s}^{(1)}+\int_{0}^{T_{2}} s^{h-1 / 2} \Delta_{s} d W_{s}^{(2)}\right]}{\left[\int_{0}^{T_{1}} s^{2 H-1} \Gamma_{s}^{2} d s+\int_{0}^{T_{2}} s^{2 h-1} \Delta_{s}^{2} d s\right]} .
$$

Let

$$
J\left(T_{1}, T_{2}\right) \equiv \int_{0}^{T_{1}} s^{2 H-1} \Gamma_{s}^{2} d s+\int_{0}^{T_{2}} s^{2 h-1} \Delta_{s}^{2} d s
$$

Then we get that

$$
J\left(T_{1}, T_{2}\right)\left(\widehat{\theta}_{T_{1}, T_{2}}-\theta\right)=-\left[\int_{0}^{T_{1}} s^{H-1 / 2} \Gamma_{s} d W_{s}^{(1)}+\int_{0}^{T_{2}} s^{h-1 / 2} \Delta_{s} d W_{s}^{(2)}\right] .
$$

Define

$$
\begin{array}{ll}
M^{(1)}(t)=\int_{0}^{t} s^{H-1 / 2} \Gamma_{s} d W_{s}^{(1)}, & 0 \leq t \leq T_{1}, \\
M^{(2)}(t)=\int_{0}^{t} s^{h-1 / 2} \Delta_{s} d W_{s}^{(2)}, & 0 \leq t \leq T_{2},
\end{array}
$$

and let $\mathbf{M}\left(T_{1}, T_{2}\right)$ be the diagonal matrix with the diagonal elements

$$
\left(M^{(1)}\left(T_{1}\right), M^{(2)}\left(T_{2}\right)\right) \text {. }
$$

Note that $M^{(i)}, i=1,2$, are independent continuous local martingales with quadratic variations

$$
\left\langle M^{(1)}\right\rangle_{t}=\int_{0}^{t} s^{2 H-1} \Gamma_{s}^{2} d s, \quad 0 \leq t \leq T_{1}
$$

and

$$
\left\langle M^{(2)}\right\rangle_{t}=\int_{0}^{t} s^{2 h-1} \Delta_{s}^{2} d s, \quad 0 \leq t \leq T_{2} .
$$

Let $\langle\mathbf{M}\rangle_{t, u}$ be a diagonal matrix with the diagonal elements $\left(\left\langle M^{(1)}\right\rangle_{t},\left\langle M^{(2)}\right\rangle_{u}\right)$. Suppose there exists a vector-valued function $\left(k_{1, t_{1}}, k_{2, t_{2}}\right)$ such that $k_{i, t_{i}}>0, i=1,2$, increasing to 
infinity as $t_{i} \rightarrow \infty$. Let $K_{t, u}$ be a diagonal matrix with the diagonal elements $\left(k_{1, t}, k_{2, u}\right)$. Suppose that

$$
K_{T_{1}, T_{2}}^{-1} \mathbf{M}\left(T_{1}, T_{2}\right) K_{T_{1}, T_{2}}^{-1} \stackrel{\mathrm{P}}{\rightarrow} \eta^{2} \quad \text { as } T_{1} \text { and } T_{2} \rightarrow \infty,
$$

where $\eta^{2}$ is a random positive diagonal matrix. Following the results in [7, Theorem 1.50] and [11, Theorem A.1], it follows that

$$
K_{T_{1}, T_{2}}^{-1}\langle\mathbf{M}\rangle_{T_{1}, T_{2}} \stackrel{\mathcal{L}}{\rightarrow} \mathbf{Z} \eta \quad \text { as } T_{1} \text { and } T_{2} \rightarrow \infty
$$

where $\mathbf{Z}$ is a diagonal matrix with the diagonal elements independent standard normal random variables and the random matrices $\mathbf{Z}$ and $\eta$ are independent. As a consequence of this result, we can give a set of sufficient conditions for asymptotic normality of the estimator $\widehat{\theta}_{T_{1}, T_{2}}$ as $T_{1} \rightarrow \infty$ and $T_{2} \rightarrow \infty$. We will now discuss a special case.

Special case. The asymptotic properties of the estimator $\widehat{\theta}_{T_{1}, T_{2}}$ depend on the processes $\Delta_{s}$ and $\Gamma_{s}$ which in turn depend on the functions $a_{1}, b_{1}, a_{2}, b_{2}$, the process $\left\{X(s), 0 \leq s \leq T_{1}\right\}$ and the process $\left\{Y(s), 0 \leq s \leq T_{2}\right\}$. However, if these functions and the processes $X$ and $Y$ are such that $\Delta_{s}=\alpha s^{1-2 h}$ and $\Gamma_{s}=\beta s^{1-2 H}$ for some constants $\alpha$ and $\beta$, then it follows that

$$
\begin{aligned}
\widehat{\theta}_{T_{1}, T_{2}}-\theta & =-\frac{\left[\int_{0}^{T_{1}} s^{H-1 / 2} \beta s^{1-2 H} d W_{s}^{(1)}+\int_{0}^{T_{2}} s^{h-1 / 2} \alpha s^{1-2 h} d W_{s}^{(2)}\right]}{\left[\int_{0}^{T_{1}} s^{2 H-1} \beta^{2} s^{2-4 H} d s+\int_{0}^{T_{2}} s^{2 h-1} \alpha^{2} s^{2-4 h} d s\right]} \\
& =-\frac{\left[\beta \int_{0}^{T_{1}} s^{1 / 2-H} d W_{s}^{(1)}+\alpha \int_{0}^{T_{2}} s^{1 / 2-h} d W_{s}^{(2)}\right]}{\left[\beta^{2} \int_{0}^{T_{1}} s^{1-2 H} d s+\alpha^{2} \int_{0}^{T_{2}} s^{1-2 h} d s\right]} \\
& =-\frac{\left[\beta \int_{0}^{T_{1}} s^{1 / 2-H} d W_{s}^{(1)}+\alpha \int_{0}^{T_{2}} s^{1 / 2-h} d W_{s}^{(2)}\right]}{\left[\beta^{2} T_{1}^{2-2 H}(2-2 H)^{-1}+\alpha^{2} T_{2}^{2-2 h}(2-2 h)^{-1}\right]} .
\end{aligned}
$$

Since the processes $W^{(1)}$ and $W^{(2)}$ are independent Wiener processes, it is easy to see that the estimator $\widehat{\theta}_{T_{1}, T_{2}}$ has a normal distribution with mean $\theta$ and variance

$$
\left[\beta^{2} T_{1}^{2-2 H}(2-2 H)^{-1}+\alpha^{2} T_{2}^{2-2 h}(2-2 h)^{-1}\right]^{-1} .
$$

It is clear that the processees $\Delta_{s}=\alpha s^{1-2 h}$ and $\Gamma_{s}=\beta s^{1-2 H}$ for some constants $\alpha$ and $\beta$ if $a_{1}=\beta b_{1}$ and $a_{2}=\alpha b_{2}$ hold.

\section{Geometric fractional Brownian motion}

We now specialize the results in Section 2 to a linear system generated by geometric fractional Brownian motions.

Let $(\Omega, \mathcal{F}, \mathrm{P})$ be a complete probability space. Consider the linear system of stochastic differential equations

$$
\begin{array}{cc}
d X(t)=\theta X(t) d t+\sigma_{1} X(t) d W^{H}(t), & X(0)=x_{0} \in \mathbb{R}, 0 \leq t \leq T_{1}, \\
d Y(t)=\theta Y(t) d t+\sigma_{2} Y(t) d W^{h}(t), & Y(0)=y_{0} \in \mathbb{R}, 0 \leq t \leq T_{2},
\end{array}
$$

defined on $(\Omega, \mathcal{F}, \mathrm{P})$, where $\left\{\theta, \sigma_{1}, \sigma_{2}\right\} \subset \mathbb{R} \backslash\{0\}$; the fractional Brownian motions

$$
\left\{W^{h}(t), 0 \leq t \leq T_{1}\right\} \quad \text { and } \quad\left\{W^{H}(t), 0 \leq t \leq T_{2}\right\}
$$

with known Hurst indices $h \in\left[\frac{1}{2}, 1\right)$ and $H \in\left[\frac{1}{2}, 1\right)$ respectively are independent. We further assume that the parameters $\sigma_{1}$ and $\sigma_{2}$ are known positive constants.

Following the notation introduced in Section 2 , let $Q$ be the product measure of the probability measures $Q^{h}$ induced on $C\left[0, T_{1}\right]$ and $Q^{H}$ induced on $C\left[0, T_{2}\right]$. With respect 
to this probability measure $Q$, the processes $\left\{\phi_{s}\right\}$ and $\left\{\eta_{s}\right\}$ chosen above should be such that the trend term should be zero in order that the process

$$
\left\{X(s), 0 \leq s \leq T_{1}\right\}
$$

be a solution of the first stochastic differential equation in the system given by (3.1) on the interval $\left[0, T_{1}\right]$ and the trend term should be zero in order that the process

$$
\left\{Y(s), 0 \leq s \leq T_{2}\right\}
$$

be a solution of the second stochastic differential equation in the system given by (3.1) on the interval $\left[0, T_{2}\right]$. Hence

$$
\sigma_{1} \int_{0}^{t} \phi_{s} d s=-\theta t, \quad 0 \leq t \leq T_{1},
$$

and

As before,

$$
\sigma_{2} \int_{0}^{t} \eta_{s} d s=-\theta t, \quad 0 \leq t \leq T_{2}
$$

$$
\begin{aligned}
\frac{d Q}{d \mathrm{P}}= & \left.\left.\frac{d Q_{H}}{d \mathrm{P}}\right|_{\mathcal{F}_{T_{1}}} \frac{d Q_{h}}{d \mathrm{P}}\right|_{\mathcal{F}_{T_{2}}} \\
= & \exp \left\{\int_{0}^{T_{1}} s^{H-1 / 2} \delta_{s} d \widetilde{W}_{s}-\frac{1}{2} \int_{0}^{T_{1}} s^{2 H-1} \delta_{s}^{2} d s\right\} \\
& \times \exp \left\{\int_{0}^{T_{2}} s^{h-1 / 2} \psi_{s} d \widehat{W}_{s}-\frac{1}{2} \int_{0}^{T_{2}} s^{2 h-1} \psi_{s}^{2} d s\right\} \\
= & \exp \left\{\int_{0}^{T_{2}} s^{h-1 / 2} \psi_{s} d \widehat{W}_{s}+\int_{0}^{T_{1}} s^{H-1 / 2} \delta_{s} d \widetilde{W}_{s}\right. \\
& \left.-\frac{1}{2}\left[\int_{0}^{T_{2}} s^{2 h-1} \psi_{s}^{2} d s+\int_{0}^{T_{1}} s^{2 H-1} \delta_{s}^{2} d s\right]\right\}
\end{aligned}
$$

Note that

$$
\delta_{t}=\left(\int_{0}^{t} k_{H}(t, s) \phi_{s} d s\right)_{t}^{\prime}=-\frac{\theta}{\sigma_{1}}\left(\int_{0}^{t} k_{H}(t, s) d s\right)_{t}^{\prime}
$$

and

$$
\psi_{t}=\left(\int_{0}^{t} k_{h}(t, s) \eta_{s} d s\right)_{t}^{\prime}=-\frac{\theta}{\sigma_{2}}\left(\int_{0}^{t} k_{h}(t, s) d s\right)_{t}^{\prime} .
$$

It is easy to see, from the computations given in [4, that

$$
\int_{0}^{t} k_{H}(t, s) d s=D_{H}^{2} t^{2-2 H}
$$

where

$$
D_{H}=\frac{C_{H}}{2 H(2-2 H)^{1 / 2}}
$$

and

$$
C_{H}=\left(\frac{2 H \Gamma\left(\frac{3}{2}-H\right)}{\Gamma\left(H+\frac{1}{2}\right) \Gamma(2-2 H)}\right)^{1 / 2}
$$

Hence

$$
\left(\int_{0}^{t} k_{H}(t, s) d s\right)_{t}^{\prime}=D_{H}^{2}(2-2 H) t^{1-2 H} .
$$


Let

It can be checked that

$$
\gamma_{H}(t)=D_{H}^{2}(2-2 H) t^{1-2 H}
$$

$$
\gamma_{H}(t)=\frac{\Gamma\left(\frac{3}{2}-H\right)}{2 H \Gamma\left(H+\frac{1}{2}\right) \Gamma(2-2 H)} t^{1-2 H}=: J_{H} t^{1-2 H} .
$$

From the above relations, we get that

$$
\begin{aligned}
\log \frac{d Q}{d \mathrm{P}}= & -\frac{\theta}{\sigma_{1}} \int_{0}^{T_{1}} s^{H-1 / 2} \gamma_{H}(s) d \widetilde{W}_{s}-\frac{\theta}{\sigma_{2}} \int_{0}^{T_{2}} s^{h-1 / 2} \gamma_{h}(s) d \widehat{W}_{s} \\
& -\frac{\theta^{2}}{2 \sigma_{1}^{2}} \int_{0}^{T_{1}} s^{2 H-1} \gamma_{H}^{2}(s) d s-\frac{\theta^{2}}{2 \sigma_{2}^{2}} \int_{0}^{T_{2}} s^{2 h-1} \gamma_{h}^{2}(s) d s .
\end{aligned}
$$

Estimation. Note that the Radon-Nikodym derivative $d Q / d \mathrm{P}$ obtained above is the Radon-Nikodym derivative of the product measure of the probability measure generated by the process $\left\{X(s), 0 \leq s \leq T_{1}\right\}$ on the space $C\left[0, T_{1}\right]$ and the probability measure generated by the independent process $\left\{Y(s), 0 \leq s \leq T_{2}\right\}$. In order to estimate the parameter $\theta$ based on the observation of the process $\left\{X(s), 0 \leq s \leq T_{1}\right\}$ and of the process $\left\{Y(s), 0 \leq s \leq T_{2}\right\}$, we maximize the likelihood function $d Q / d \mathrm{P}$ or equivalently $\log (d Q / d \mathrm{P})$. Differentiating the function $\log (d Q / d \mathrm{P})$ with respect to $\theta$ and equating the derivative to zero, we obtain the likelihood equation

$$
\theta\left[\frac{J_{H}^{2}}{\sigma_{1}^{2}} \frac{T_{1}^{2-2 H}}{2-2 H}+\frac{J_{h}^{2}}{\sigma_{2}^{2}} \frac{T_{2}^{2-2 h}}{2-2 h}\right]=-\left[\frac{J_{H}}{\sigma_{1}} \int_{0}^{T_{1}} s^{1 / 2-H} d \widetilde{W}_{s}+\frac{J_{h}}{\sigma_{2}} \int_{0}^{T_{2}} s^{1 / 2-h} d \widehat{W}_{s}\right],
$$

which leads to the estimator, hereafter called the maximum likelihood estimator (MLE), given by

$$
\widehat{\theta}_{T_{1}, T_{2}}=-\frac{\left[\frac{J_{H}}{\sigma_{1}} \int_{0}^{T_{1}} s^{1 / 2-H} d \widetilde{W}_{s}+\frac{J_{h}}{\sigma_{2}} \int_{0}^{T_{2}} s^{1 / 2-h} d \widehat{W}_{s}\right]}{\left[\frac{J_{H}^{2}}{\sigma_{1}^{2}} \frac{T_{1}^{2-2 H}}{2-2 H}+\frac{J_{h}^{2}}{\sigma_{2}^{2}} \frac{T_{2}^{2-2 h}}{2-2 h}\right]} .
$$

It can be checked that (see [3, Eq. (14)])

$$
d \widetilde{W}_{s}=d W_{s}^{(1)}-\frac{\theta}{\sigma_{1}} s^{H-1 / 2} \gamma_{H}(s) d s
$$

and

$$
d \widehat{W}_{s}=d W_{s}^{(2)}-\frac{\theta}{\sigma_{2}} s^{h-1 / 2} \gamma_{h}(s) d s,
$$

where $W^{(1)}$ and $W^{(2)}$ are independent Wiener processes under the measure $Q$. Using these relations, it follows that

$$
\widehat{\theta}_{T_{1}, T_{2}}-\theta=-\frac{\left[\frac{J_{H}}{\sigma_{1}} \int_{0}^{T_{1}} s^{1 / 2-H} d W_{s}^{(1)}+\frac{J_{h}}{\sigma_{2}} \int_{0}^{T_{2}} s^{1 / 2-h} d W_{s}^{(2)}\right]}{\left[\frac{J_{H}^{2}}{\sigma_{1}^{2}} \frac{T_{1}^{2-2 H}}{2-2 H}+\frac{J_{h}^{2}}{\sigma_{2}^{2}} \frac{T_{2}^{2-2 h}}{2-2 h}\right]} .
$$

In particular, it follows that the estimator $\widehat{\theta}_{T_{1}, T_{2}}-\theta$ has the normal distribution with mean zero and variance

$$
\left[\frac{J_{H}^{2}}{\sigma_{1}^{2}} \frac{T_{1}^{2-2 H}}{2-2 H}+\frac{J_{h}^{2}}{\sigma_{2}^{2}} \frac{T_{2}^{2-2 h}}{2-2 h}\right]^{-1}
$$

Suppose $h \geq H$. Further suppose that we observe the process $X$ governed by the first equation in the system up to time $T_{1}=T$ and observe the process $Y$ governed by the second equation in the system up to time

$$
T_{2}=T^{\frac{1-H}{1-h}} .
$$


Then the variance of the MLE is given by

$$
\left[\frac{J_{H}^{2}}{\sigma_{1}^{2}} \frac{T_{1}^{2-2 H}}{2-2 H}+\frac{J_{h}^{2}}{\sigma_{2}^{2}} \frac{T_{2}^{2-2 h}}{2-2 h}\right]^{-1},
$$

which is of the order $O\left(T^{2 H-2}\right)$. A better estimator with smaller variance can be obtained by suitably choosing $T_{1}=T$ and

$$
T_{2}=c T^{\frac{1-H}{1-h}}
$$

where $c$ is defined by the relation

$$
\frac{J_{H}^{2}}{\sigma_{1}^{2}} \frac{T^{2-2 H}}{2-2 H}=c^{2-2 h} \frac{J_{h}^{2}}{\sigma_{2}^{2}} \frac{T_{2}^{2-2 h}}{2-2 h} .
$$

Remarks. The methods of this paper can be extended to study the problem of estimation of the parameter $\theta$ for more general linear systems of the type

$$
\begin{gathered}
d X_{i}(t)=\left[\theta a_{i}\left(t, X_{i}(t)\right)+c_{i}\left(t, X_{i}(t)\right)\right] d t+b_{i}\left(t, X_{i}(t)\right) d W^{H_{i}}(t), \\
0 \leq t \leq T_{i}, \quad 1 \leq i \leq n .
\end{gathered}
$$

\section{BIBLIOGRAPHY}

1. M. L. Kleptsyna and A. Le Breton, Statistical analysis of the fractional Ornstein-Uhlenbeck type process, Statist. Inf. Stochast. Proces. 5 (2002), 229-248. MR1943832 (2003i:62140)

2. R. Liptser, A strong law of large numbers for local martingales, Stochastics, 3 (1980), 217-228. MR.573205 (83d:60057)

3. Y. Mishura and N. Rudomino-Dusyatska, Consistency of Drift Parameter Estimates in Fractional Brownian Diffusion Models, Research paper No. 04-2004, Acturial Education and Reference Centre of the Ukrainian Acturial Society, Lviv, Ukraine, 2004.

4. I. Norros, E. Valkeila, and J. Virtamo, An elementary approach to a Girsanov formula and other analytical results on fractional Brownian motion, Bernoulli, 55 (1999), 571-587. MR1704556 (2000f:60053)

5. D. Nualart and A. Rascanu, Differential Equations Driven by Fractional Brownian Motion, Preprint, 2001. MR1893308 (2003f:60105)

6. B. L. S. Prakasa Rao, Statistical Inference for Diffusion Type Processes, Arnold, London, and Oxford University Press, New York, 1999a. MR1717690 (2000k:62149)

7. B. L. S. Prakasa Rao, Semimartingales and their Statistical Inference, CRC Press, Boca Raton and Chapman and Hall, London, 1999b. MR1689166 (2000h:62004)

8. B. L. S. Prakasa Rao, Parametric estimation for linear stochastic differential equations driven by fractional Brownian motion, Random Operators and Stochastic Equations, 11 (2003), 229242. MR2009183 (2005a:62188)

9. B. L. S. Prakasa Rao, Berry-Esseen bound for MLE for linear stochastic differential equations driven by fractional Brownian motion, Journal of Korean Statistical Society, 34 (2005), 281295. MR:2233242 (2007m:62128)

10. B. L. S. Prakasa Rao, Instrumental variable estimation for linear stochastic differential equations driven by fractional Brownian motion, Stochastic Analysis and Applications 25 (2007), no. 6, 1203-1215. MR.2367310 (2009b:62169)

11. M. Sorensen, Likelihood methods for diffusions with jumps, Statistical Inference in Stochastic Processes (N. U. Prabhu and I. V. Basawa, eds.), Marcel Dekker, New York, 1991, 67-105. MR.1138259 (92k:62144)

12. M. Zähle, Integration with respect to fractional functions and stochastic calculus I, Prob. Theory Relat. Fields, 111 (1998), 333-374. MR 1640795 (99j:60073)

University of Hyderabad, Hyderabad 500 046, India

E-mail address: blsprao@gmail.com

Received 30/AUG/2007

Originally published in English 